\title{
Expanding a portfolio of (FO-) SPR surface chemistries with the Co(III)- NTA oriented immobilization of His6-tagged bioreceptors for applications in complex matrices
}

\author{
Jia-Huan Qu†, Sara Horta , Filip Delport§, Machteld Sillen", Nick Geukens ${ }^{\perp}$, Da-Wen Sun ${ }^{\nabla}$, Karen \\ Vanhoorelbeke ${ }^{\ddagger}$ Paul Declerck॥, Jeroen Lammertyn ${ }^{\dagger *}$, and Dragana Spasic ${ }^{\dagger}$
}

†Department of Biosystems, Biosensors Group, KU Leuven, Willem de Croylaan 42, 3001 Leuven, Belgium

‡Laboratory for Thrombosis Research, IRF Life Sciences, KU Leuven Campus Kulak, Kortrijk, Belgium

§FOx Biosystems, Bioville, Agoralaan Abis, 3590 Diepenbeek, Belgium

"Department of Pharmaceutical and Pharmacological Sciences, Laboratory for Therapeutic and Diagnostic Antibodies, KU Leuven, O\&N II Herestraat 49,3000 Leuven, Belgium

${ }^{\perp}$ PharmAbs, KU Leuven, Herestraat 49, box 820, B 3000, Leuven, Belgium
${ }^{\nabla}$ School of Food Science and Engineering, South China University of Technology, Guangzhou 510641, China

KEYWORDS: nitrilotriacetic acid (NTA), fiber optic surface plasmon resonance (FO-SPR), label-free bioassay, sandwich bioassay, plasminogen activator inhibitor-1 (PAI-1), bioreceptor orientation

\begin{abstract}
Cobalt-nitrilotriacetic acid (Co(III)-NTA) chemistry is a recognized approach for oriented patterning of His6tagged bioreceptors. We have applied the matching strategy for the first time on an SPR platform, namely the commercialized FO-SPR. To accomplish this, His6-tagged bioreceptor (scFv-33H1F7) and its target PAI-1 were used as a model system, after scrutinizing the specificity of their interaction. When benchmarked to traditional carboxyl based selfassembled monolayers (SAM), NTA allowed (1) more efficient FO-SPR surface coverage with bioreceptors compared to the former and (2) realization of thus far difficult-to-attain label-free bioassays on the FO-SPR platform in both buffer and 20fold diluted human plasma. Moreover, Co(III)-NTA surface proved to be compatible with traditional gold nanoparticlemediated signal amplification in the buffer as well as in 10-fold diluted human plasma, thus expanding the dynamic detection range to low ng/mL. Both types of bioassays revealed that scFv-33H1F7 immobilized on the FO-SPR surface using different concentrations $(20,10$ or $5 \mu \mathrm{g} / \mathrm{mL})$ had no impact on the bioassay sensitivity, accuracy or reproducibility despite the lowest concentration effectively resulting in close to $20 \%$ fewer bioreceptors. Collectively, these results highlight the importance of Co(III)-NTA promoting the oriented patterning of bioreceptors on the FO-SPR sensor surface for securing robust and sensitive bioassays in complex matrices, both in label-free and labelled formats.
\end{abstract}

In the last three decades, significant innovations have been made in the field of surface plasmon resonance (SPR) biosensing, leading to the creation of robust, specific and sensitive platforms used for monitoring/quantification of biomolecular interactions in real-time. In this context, surface functionalization of the sensing platform has played a vital role. Generally, antibodies, ${ }^{1}$ nanobodies, ${ }^{2}$ nucleic acids, ${ }^{3}$ or other recognition molecules (e.g. molecularly imprinted polymers, ${ }^{4}$ enzymes or even whole cells ${ }^{5}$ ) are used as bioreceptors to recognize the target, and are often immobilized on the sensor surface through different types of self-assembled monolayers (SAMs) attached to the gold surface via thiol bonds. One of the most commonly used SAMs for antibody immobilization is carboxylic acid ( $\mathrm{COOH})$ SAM that forms covalent amide bonds with bioreceptors. ${ }^{1,6}$ However, antibodies immobilized in such a way have a random orientation resulting in a reduction of available binding sites and induced steric hindrance. ${ }^{7}$
To realize better control over the surface functionalization, nitrilotriacetic acid (NTA) SAMs have been broadly reported for immobilization of hexahistidine (His6)-tagged biomolecules in a site-specific and oriented manner. ${ }^{8-11}$ Originally, NTA has been developed for purification of His6tagged proteins, as it can form chelates with bivalent metal cations (e.g., $\mathrm{Cu}^{2+}, \mathrm{Ni}^{2+}, \mathrm{Zn}^{2+}, \mathrm{Co}^{2+}$ ) through four sites of the octahedral coordination sphere, leaving two sites for binding the imidazole groups in two of the six consecutive histidine residues of His6-tagged proteins. ${ }^{12}$ Among all metal cations, $\mathrm{Ni}^{2+}$ and $\mathrm{Zn}^{2+}$ have been most frequently used both for protein purification and sensor surface functionalization as their chelates represent a good compromise in terms of affinity $\left(\mathrm{Cu}^{2+}>\mathrm{Ni}^{2+}>\mathrm{Zn}^{2+} \geq \mathrm{Co}^{2+}\right)$ and specificity $\left(\mathrm{Co}^{2+}>\mathrm{Zn}^{2+}>\mathrm{Ni}^{2+}>\mathrm{Cu}^{2+}\right) \cdot{ }^{13-19}$ In the context of biosensing, Ni(II)-NTA has been employed frequently to pattern His6-tagged bioreceptors on sensor surfaces. ${ }^{20-22}$ Specifically, COOH and NTA SAMs were compared using a quartz crystal microbalance (QCM) biosensor, which 
revealed that the $\mathrm{Ni}(\mathrm{II})-\mathrm{NTA}$ surface exhibited a higher immobilization capacity and binding activity for bioreceptors than the $\mathrm{COOH}$ surface, enabling a higher detection sensitivity ${ }^{22}$. Additionally, the NTA surface allowed an optional regeneration process either to remove the His6-tagged bioreceptors or only the analyte, for the next cycle of specific detection, enabling a reusable and low-cost sensing system. ${ }^{22}$ However, as the His6-tagged proteins were immobilized on $\mathrm{Ni}(\mathrm{II})$-NTA surface via coordinate covalent bond, the sensing surface was unstable with slow and continuous dissociation of immobilized proteins, ${ }^{23-25}$ especially in complex matrices with low $\mathrm{pH}$, reducing agents, chelators like ethylenediaminetetraacetic acid (EDTA) and imidazole, or other molecules competing with imidazole groups in His6-tags. ${ }^{26}$
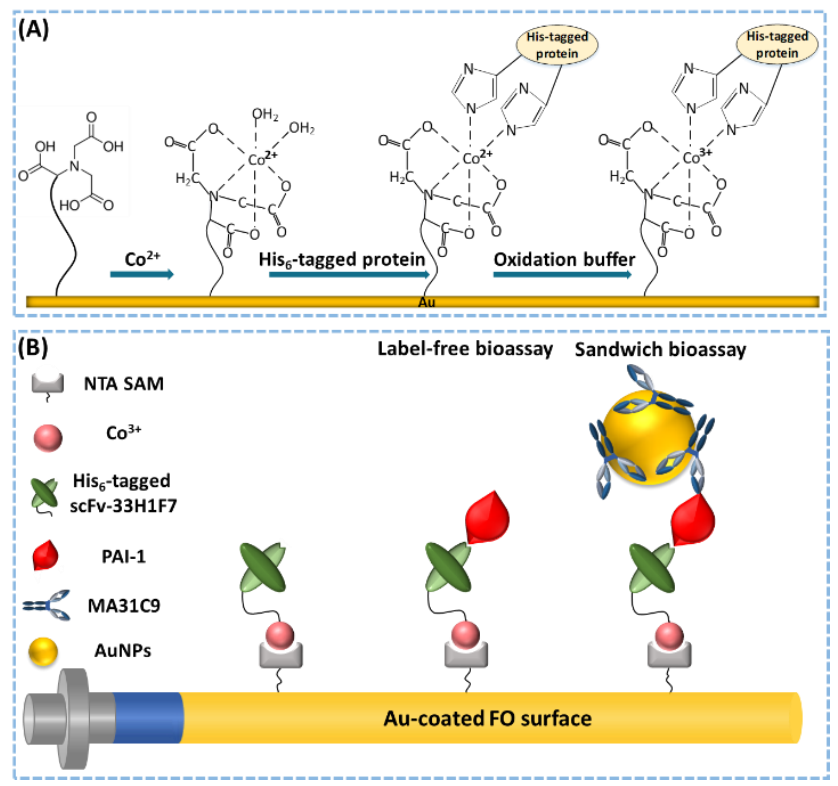

Figure 1. Schematic representation of A) the immobilization steps of His6-tagged protein on Au-coated surface by Co(III)NTA strategy, and B) FO-SPR based bioassay of PAI-1 using Co(III)-NTA for bioreceptor immobilization.

To improve NTA-based surface stability in complex biological environments, two types of solutions have been proposed. One solution was adopting molecular or surface multivalency to achieve more stable interaction towards His $_{6}$-tagged proteins. For example, multivalent chelators, i.e. bis-NTA and tris-NTA, proved to achieve higher affinity towards conventional His6-tagged proteins compared to mono-NTA due to molecular multivalency. ${ }^{27-29}$ A higher density of chelators as binding sites also resulted in a more stable attachment of His6-tagged proteins due to the effect of surface multivalency. ${ }^{27} \mathrm{~A}$ second proposed solution was to use double-His6-tagged proteins separated by an 11amino acid spacer, which proved to be stronger in binding to Ni(II)-mono-NTA than single-His6-tagged proteins or proteins with two single-His 6 -tags at two different ends. ${ }^{9}$ Here, additionally, the formation of covalent bonds (e.g. via amine coupling reaction) was performed after NTA based immobilization to achieve cross-linking and further enhance the interaction. ${ }^{25,30}$ However, to apply the aforementioned solutions, either a more complicated NTA surface was required or the His 6 -tagged protein had to be additionally engineered during production.

To overcome these limitations, Wegner's group proposed to introduce $\mathrm{Co}^{3+}$ as the mediator ion between NTA and His 6 -tagged proteins, which creates a more stable and inert functionalized surface. ${ }^{26,31,32}$ Compared with $\mathrm{Co}^{2+}$ and $\mathrm{Ni}^{2+}$ complexes, the $\mathrm{Co}^{3+}$ complex exhibited substantially higher association and lower dissociation rate constants (about 20 and 12 orders of magnitudes, respectively),,33-35 without possibility to regenerate the surface due to very slow ligand exchange. The Co(III)-NTA chemistry was applied and validated in the development of several different biosensing platforms, including $\mathrm{QCM}^{32}$ biolayer interferometry, ${ }^{36}$ and fluorescent sensors. ${ }^{37}$ Not limited to NTA as the chelating agent, plant polyphenolic tannic acid (TA) was also employed to chelate $\mathrm{Co}^{2+} / \mathrm{Co}^{3+}$ and immobilize His6-tagged proteins for QCM measurements. However, a Co(III)-NTA functionalized surface has not been explored for any SPR biosensors to date.

In the current work, we investigate for the first time the possibility to immobilize His6-tagged bioreceptors on the FO-SPR platform using the Co(III)-NTA strategy, next to testing the immobilization efficiency of bioreceptors and surface coverage. Moreover, we study the effect this surface chemistry has on several aspects of the FO-SPR sensing performance, including (1) the achievable sensitivity of both label-free and labelled bioassays, (2) reproducibility of bioassays in buffer as well as in more complex matrices, such as plasma, (3) stability and robustness of the Co(III)-NTA-based surface when exposed to plasma matrix, and, (4) specificity of the bioassay. To examine this, plasminogen activator inhibitor-1 (PAI-1) is used here, solely as a model system, with anti-PAI-1 specific bioreceptors: His6-tagged anti-PAI-1 antibody fragment (scFv-33H1F7) as capture and anti-PAI-1 monoclonal antibody (MA-31C9) as a detection antibody, the latter being immobilized on gold nanoparticles (AuNPs) for signal amplification. This set of reagents is selected as they are all in-house generated and previously wellcharacterized, assuring their effective performance in this study. The immobilization of scFv-33H1F7 at different concentrations is first studied in terms of both signal shift and kinetics. In order to test the validity of the selected model system, the specific binding between scFv-33H1F7 and PAI-1 is characterized in detail. Label-free bioassays of PAI-1 are developed in buffer followed by testing the surface stability and bioassay performance in 20-fold diluted human plasma. Lastly, the Co(III)-NTA surface is tested for its compatibility with the AuNPs-mediated signal amplification strategy in both buffer and 10-fold diluted plasma towards achieving more sensitive sandwich bioassays. Calibration curves are established for both labelfree and sandwich bioassays to evaluate the sensing performance. The concept of the applied study is schematically depicted in Figure 1. 


\section{EXPERIMENTAL SECTION}

\section{Reagents and buffers}

Trizma base, imidazole, $\mathrm{H}_{2} \mathrm{O}_{2}$, phosphate-buffered saline (PBS) and albumin from bovine serum (BSA) were purchased from Sigma-Aldrich (Diegem, Belgium). $\mathrm{CoCl}_{2} \cdot 6 \mathrm{H}_{2} \mathrm{O}$ was provided by Acros Organics (Geel, Belgium). NaCl, ethanol, and SuperBlock (PBS) blocking buffer were obtained from Thermo Fisher Scientific (Erembodegem, Belgium). Tween 20 was purchased from AppliChem GmbH (Darmstadt, Germany). Nitrilotriacetic acid (NTA) SAM formation reagent was acquired from Dojindo Laboratories (Kumamoto, Japan). Gold nanoparticles (AuNPs) EM.GC20 were obtained from BBI Solutions (Cardiff, U.K) with an average diameter of $20 \mathrm{~nm}$. The following biological reagents were all produced in Laboratory of Therapeutic and Diagnostic Antibodies (KU Leuven, Belgium), as described in the previous publications: human-derived PAI-1 purified from E. coli, ${ }^{38}$ anti-PAI-1 monoclonal antibody (mAb) MA-31C9, ${ }^{39}$ His6-tagged single-chain variable fragment of mAb MA-33H1F7 (scFv33H1F7), and PAI-1 depleted, pooled normal human plasma $^{40}$ with residual endogenous PAI-1 ranging from 1.5 to $3.6 \mathrm{ng} / \mathrm{mL}$. MDTCS (N-terminal portion of ADAMTS13) 41 with His $_{6}$-tag was produced as previously described. ${ }^{42}$ Cloned human anti-MDTCS monoclonal antibody II-1 (specifically recognizing the $\mathrm{S}$ (spacer) domain in MDTCS) was generated as previously described. ${ }^{43}$ Control IgG was purchased from Thermo Fisher Scientific (USA). The following buffers have been used in this work: (1) Tris-buffered saline, TBS $(50 \mathrm{mM}$ Tris- $\mathrm{HCl}, 300 \mathrm{mM} \mathrm{NaCl}, \mathrm{pH} 8.0$ prepared at controlled temperature of $\left.25^{\circ} \mathrm{C}\right)$; (2) imidazole buffer $(100 \mathrm{mM}$ imidazole in TBS, $\mathrm{pH} 8.0$ prepared at controlled temperature of $\left.25^{\circ} \mathrm{C}\right)$; (3) oxidation buffer $\left(50 \mathrm{mM} \mathrm{H} \mathrm{H}_{2} \mathrm{O}_{2}\right.$ diluted in PBS pH 6.5) ${ }^{26}$ and (4) detection buffer (10 mM PBS pH 7.4, 0.01\% Tween 20). PBS at pH 6.5 was selected as oxidation buffer to promote the stability of $\mathrm{H}_{2} \mathrm{O}_{2}$ at acidic condition while preventing the protonation of imidazole ring in His $_{6}$-tag that can happen below $\mathrm{pH} 6.0 .^{44}$ All the buffer solutions were made using ultrapure water from a Millipore Synergy UV water purifying system (Millipore SAS, Molsheim, France).

\section{FO-SPR sensing platform and functionalization of the FO sensor surface}

The FO-SPR biosensor commercialized by FOx Biosystems (Diepenbeek, Belgium) has been elaborately described in Supporting Information (Figure S1) and it is based on the previously published, in-house developed SPR platform. ${ }^{1,6}$ A detailed description of the manufacturing process for gold-coated FO probes can be found in our previous publications. ${ }^{1,45}$ The gold-coated FO probes were fully immersed in $0.2 \mathrm{mM}$ NTA SAM solution (dissolved in ethanol) and incubated at $4{ }^{\circ} \mathrm{C}$ overnight. Before use, the NTA functionalized FO probes were rinsed with ethanol followed by TBS buffer for stabilization. Afterward, the NTA molecules on the surface of the FO probes were activated by immersion in $100 \mathrm{mM} \mathrm{CoCl} \mathrm{m}_{2}$ solution dissolved by ultrapure water for $10 \mathrm{~min}$ to form the Co(II)-NTA chelate. Then, the His6-tagged scFv-33H1F7 bioreceptors, diluted in TBS $(20,10$ and $5 \mu \mathrm{g} / \mathrm{mL})$ with $0.01 \%$ Tween 20 , were immobilized on the Co(II)-NTA surface by immersion for $30 \mathrm{~min}$. Subsequently, the $\mathrm{Co}^{2+}$ core was oxidized to $\mathrm{Co}^{3+}$ by oxidation buffer for $30 \mathrm{~min}$. Then the $\mathrm{FO}$ probes were immersed in imidazole buffer for 2 min to displace the instable chelate structures, followed by oxidation buffer for 10 min to further consolidate the NTA surface. As demonstrated with the sensorgram (Figure S2), no His6tagged scFv-33H1F was removed by imidazole, proving successful oxidation of $\mathrm{Co}^{2+}$ to $\mathrm{Co}^{3+}$. The functionalized sensor surface was finally blocked by SuperBlock (PBS) blocking buffer for 12 min and stabilized by the detection buffer for $4 \mathrm{~min}$ before quantification of PAI-1. Each functionalized FO probe was used for the measurement of a single concentration, which applies to the entire study. The whole bioassay was performed at a controlled temperature of $25^{\circ} \mathrm{C}$.

\section{Label-free bioassay of PAl-1 in buffer and diluted plasma}

For developing a label-free bioassay of PAI-1 in the buffer, scFv-33H1F7 bioreceptors were immobilized on the FO probes at concentrations of 20,10 or $5 \mu \mathrm{g} / \mathrm{mL}$. Regarding 5 $\mu \mathrm{g} / \mathrm{mL}$, scFv-33H1F7 was pre-mixed with $5 \mu \mathrm{g} / \mathrm{mL}$ of MDTCS to form a 1:1 (concentration ratio) solution for functionalization (for more details, please see section RESULTS AND DISCUSSION). After being fully functionalized, the FO probes were immersed in the detection buffer for $30 \mathrm{~min}$, spiked with PAI-1 $(0.25,0.5,1$, 2,4 and $8 \mu \mathrm{g} / \mathrm{mL}$ ). Buffer sample without PAI-1 was used as a negative control. The calibration curves were made for all three scFv-33H1F7 concentrations $\left(\mathrm{n}_{\mathrm{s}}=2\right.$ for each condition). For the implementation of the label-free bioassay in a complex matrix, the FO probes functionalized with $10 \mu \mathrm{g} / \mathrm{mL}$ of scFv-33H1F7 were first immersed in 10, 20, 50 and 100-fold diluted human plasma without spiked PAI-1 (dilutions of plasma were performed using detection buffer). Based on these results and to further quantify PAI1 , the functionalized FO probes $(10 \mu \mathrm{g} / \mathrm{mL}$ of scFv-33H1F7) were immersed in 20 -fold diluted human plasma, spiked with PAI-1 $(0.25,0.5,1,2,4$ and $8 \mu \mathrm{g} / \mathrm{mL})$ and data were used to generate the calibration curve $\left(\mathrm{n}_{\mathrm{s}}=4\right.$ for each concentration of PAI-1, performed with FO probes functionalized in two independent batches). Plasma used in this paper was pooled normal human plasma that was PAI1 depleted, thereby having residual endogenous PAI-1 ranging from 1.5 to $3.6 \mathrm{ng} / \mathrm{mL}^{40}$

\section{Sandwich bioassay of PAl-1 in the buffer and diluted plasma}

For detecting low amounts of PAI-1 using a sandwich bioassay, the FO probes were first functionalized with scFv-33H1F7 bioreceptors by dipping them in concentrations of 20,10 and $5 \mu \mathrm{g} / \mathrm{mL}$. The functionalized FO probes were immersed in the detection buffer spiked with PAI-1 for $30 \mathrm{~min}$, followed by a washing step using the detection buffer for $1 \mathrm{~min}$. Then the FO probe was transferred to $0.5 \%$ BSA in PBS for 4 min followed by the signal amplification with functionalized AuNPs for 30 min. Finally, the FO probe was washed by $0.5 \%$ BSA in PBS for 1 min. The AuNPs were conjugated with MA-31C9 at the final 
concentration of $5 \mu \mathrm{g} / \mathrm{mL}$ in the AuNPs solution, following the protocol as described in our previous publication ${ }^{6}$. The optical density value of the functionalized AuNPs was adjusted to 0.5 using a spectrophotometer (SpectraMax iD3, Molecular devices, San Jose, California, U.S.A) before use. Calibration curves were made with PAI-1 concentrations of $0,3.125,6.25,12.5,25,50,100,200$ and $400 \mathrm{ng} / \mathrm{mL}$ in the detection buffer using FO probes dipped in 20, 10 and 5 $\mu \mathrm{g} / \mathrm{mL}$ of scFv-33H1F7 concentrations (with $\mathrm{n}_{\mathrm{s}}=2$ for each). For the implementation of the sandwich bioassay in a complex matrix, the FO probe was functionalized with 10 $\mu \mathrm{g} / \mathrm{mL}$ of scFv-33H1F7. Following the same procedure as in buffer, the functionalized FO probe was finally immersed in 10-fold diluted plasma with PAI-1 ranging from 0 - 400 $\mathrm{ng} / \mathrm{mL}$. The obtained data were used to generate the calibration curve $\left(n_{s}=4\right.$ for each concentration of PAI-1, performed with functionalized FO probes and conjugated AuNPs prepared in two independent batches).

\section{Data analysis}

FO-SPR data were recorded with FOx software (FOx Biosystems) and further processed using MATLAB 2015b (The MathWorks Inc., Natick). All the calculations and graphs throughout the paper were obtained by programming in MATLAB. The following equation for nonlinear regression applies to the whole study.

As displayed below, the calibration curves were fitted via Eq 1 for non-linear (one-site binding) regression.

$y=x * A /(x+B)$

where $x$ represents the PAI- 1 concentration, $y$ represents the SPR shift, and $A$ and $B$ represent the fitting parameters.

To compare the sensitivity of the different bioassays developed at different conditions, the limit of detection (LOD) was calculated based on a statistically robust method. ${ }^{46}$ Using this method, the standard deviations of both blank samples and test samples were taken into account to calculate the LOD estimates. Unlike the fourparameter logistic curve fit used in the corresponding paper, ${ }^{46}$ here we used a one-site binding curve fit (Eq 1) for determining PAI-1 concentrations based on the measured SPR signal. Lastly, LOD estimates for different bioassays were generated at a 95\% confidence interval, which were further compared using Welch's $t$-test to calculate a pvalue and statistically determine the significant difference between every two bioassays. The calculation of LOD estimates was realized by programming in MATLAB based on the codes provided in the supporting information of the corresponding paper ${ }^{46}$ with our own adjustment for this case.

\section{RESULTS AND DISCUSSION}

Immobilization of $\mathrm{His}_{6}$-tagged scFv-33H1F7 bioreceptors on the FO probe surface

Because the Co(III)-NTA strategy has not been previously implemented on (FO) SPR sensor surfaces, we first wanted to test the efficiency of immobilizing bioreceptors using this surface chemistry. Therefore, His6-tagged scFv33H1F7 bioreceptors were immobilized using three different concentrations $(20,10$ and $5 \mu \mathrm{g} / \mathrm{mL})$ following the procedure as explained in the EXPERIMENTAL SECTION. A bioreceptor concentration of $20 \mu \mathrm{g} / \mathrm{mL}$ was selected based on our previously optimized conditions when using $\mathrm{COOH}$ SAM. ${ }^{6}$ This concentration was also further reduced (2-fold and 4-fold) in order to test whether NTA-promoted orientation of bioreceptors can compensate for the effectively lower number of bioreceptors on the surface. As displayed in Figure 2, similar SPR shifts of 10.6 $\mathrm{nm}$ in $30 \mathrm{~min}$ were observed for 20 and $10 \mu \mathrm{g} / \mathrm{mL}$ of scFv33H1F7, respectively (Figure 2A), suggesting that in both cases a comparable number of bioreceptors was immobilized on the FO probes with the only noticeable difference in the immobilization kinetics (Figure 2B). These obtained SPR shifts were significantly higher compared to the $7.5 \mathrm{~nm}$ shifts previously observed for $\mathrm{COOH}$ SAM mediated immobilization of $20 \mu \mathrm{g} / \mathrm{mL}$ of bioreceptors. ${ }^{6}$ This difference in SPR shifts extrapolates in a larger number of bioreceptors on the NTA-surface compared to the $\mathrm{COOH}$ SAM, which might be even more pronounced when taking into account that scFv-33H1F7 (scFv) (used in this study) is of a smaller size compared to the previously used full-size IgG antibodies (molecular weight (MW) of approx. $38 \mathrm{kDa}$ and $150 \mathrm{kDa}$, respectively). To further prove this, we have calculated bioreceptor density starting from the weight sensitivity of the FO-SPR sensors previously established in our group, ${ }^{47}$ i.e., $1 \mathrm{~nm}$ shift representing the binding of $0.286 \mathrm{ng}$ bioanalyte per $\mathrm{mm}^{2}$, assuming the molecular density is constant and the distance effect can be neglected. Using this, together with the MW of two bioreceptors and measured FO-SPR shift for their immobilization at $20 \mu \mathrm{g} / \mathrm{mL}$, the actual densities of His 6 -tagged scFv-33H1F7 and full IgG were estimated as $7.98 \times 10^{-8} \mathrm{~mol} / \mathrm{m}^{2}$ and $1.43 \times 10^{-8} \mathrm{~mol} / \mathrm{m}^{2}$, respectively. Collectively, these data suggest that NTA-mediated immobilization of bioreceptors on the FO-SPR surface might enable more efficient surface coverage, probably due to the better orientation of His ${ }_{6}$-tagged bioreceptors, as previously reported, and thus improved surface homogeneity.48,49 Importantly, because no significant difference was observed in the number of bioreceptors after 30 min immobilization when using 10 or $20 \mu \mathrm{g} / \mathrm{mL}$ of scFv-33H1F7, the former can be regarded as sufficient to ensure a high sensing performance while also saving biological reagents. Interestingly, decreasing the scFv$33 \mathrm{H} 1 \mathrm{~F} 7$ concentration to $5 \mu \mathrm{g} / \mathrm{mL}$ resulted in a final immobilization shift that was still about $80 \%$ of the signal obtained for 10 or $20 \mu \mathrm{g} / \mathrm{mL}$ of scFv-33H1F7, despite evidently slower immobilization kinetics. 

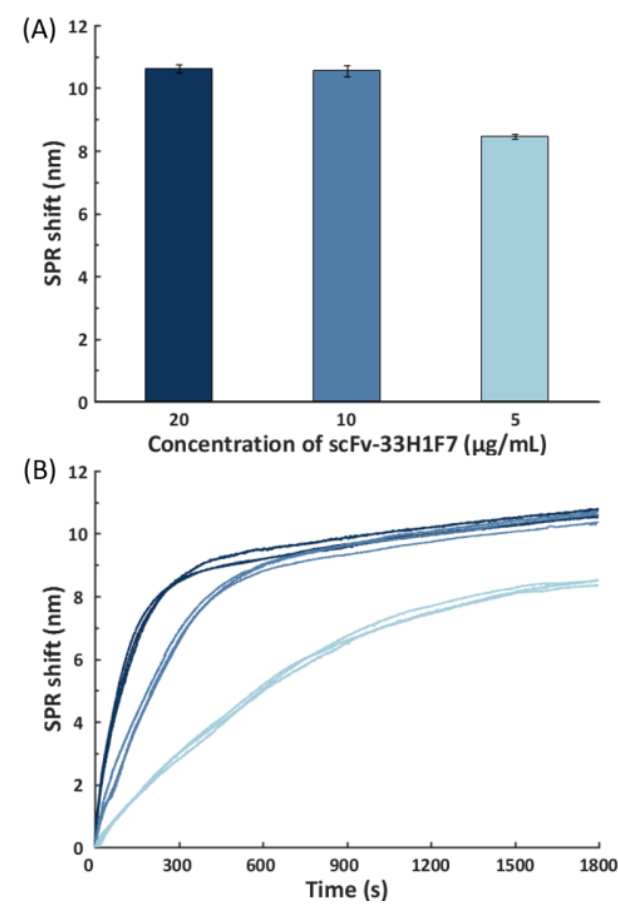

Figure 2. Immobilization of His6-tagged scFv-33H1F7 bioreceptors at different concentrations $(20,10$ and $5 \mu \mathrm{g} / \mathrm{mL})$. (A) SPR signal shift and (B) kinetic curves (three curves are depicted for each scFv-33H1F7 concentration). Error bars represent standard deviations $\left(\mathrm{n}_{\mathrm{s}}=3\right)$.

\section{Characterization of PAI-1 as a model system}

In order to demonstrate the validity of PAI-1 (MW of approx. $45 \mathrm{kDa}$ ) as the selected model system, we first tested the specificity of the interaction between $\mathrm{scFv}$ 33H1F7 and PAI-1 (Figure 3). To do that, we have prepared three types of FO probes functionalized with: (1) $10 \mu \mathrm{g} / \mathrm{mL}$ of His6-tagged scFv-33H1F7 bioreceptors, (2) $10 \mu \mathrm{g} / \mathrm{mL}$ of His6-tagged MDTCS bioreceptors, which specifically bind to their target (mAb II-1) but should not interact with PAI-1 (this bioreceptor-target pair was also selected as in-house generated and well-characterized set of reagents, serving purely as control) and (3) Co(III)-NTA SAM without any bioreceptors. Each of these FO probes was then used for label-free detection of four different targets, i.e. PAI-1, II-1, IgG and BSA, at the concentration of $1 \mu \mathrm{g} / \mathrm{mL}$ in detection buffer. FO probes with scFv-33H1F7 only interacted with PAI-1, unlike other tested targets, proving the specificity of the scFv-33H1F7/PAI-1 interaction. Similar results were obtained for the MDTCS/II-1 bioreceptor-target pair, which additionally indicated that PAI-1 does not bind nonspecifically when other bioreceptors were immobilized on the FO probe. Intriguingly, using the FO probe without any bioreceptors revealed that PAI-1 was not only binding to the surface but also that the obtained SPR shift was very similar to the one obtained when scFv-33H1F7 bioreceptor was present. ${ }^{50,51}$ This was, however, not observed for any of the other three molecules tested.

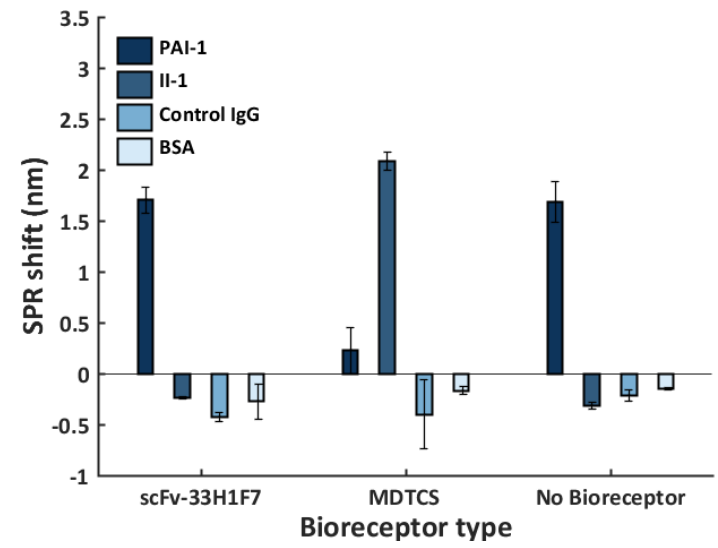

Figure 3. Functionalization of the FO probes with $10 \mu \mathrm{g} / \mathrm{mL}$ of scFv-33H1F7 or MDTCS as well as without any bioreceptors for label-free detection of four different molecules at $1 \mu \mathrm{g} / \mathrm{mL}$, i.e. PAI-1 (target of scFv-33H1F7), II-1 (target of MDTCS), control IgG and BSA (the latter two are used as controls as no binding is expected to either scFv-33H1F7 or MDTCS). The observed negative shifts of $<0.5 \mathrm{~nm}$ correspond to the minor signal instability due to the incomplete washing step after blocking with SuperBlock buffer. Error bars represent standard deviations $\left(\mathrm{n}_{\mathrm{s}}=2\right)$.

To further understand why PAI-1 was binding to the NTA surface without bioreceptors, we looked into the amino acid sequence of the PAI- 1 molecule. As revealed by Figure $4 \mathrm{~A}$ and Figure S3A, PAI-1 contains two consecutive histidine amino acids, which are exposed to the surface according to the 3-dimensional (3D) structure and as such might interact with the free binding sites in Co(II)-NTA chelate. This hypothesis was further proven by performing three different experiments. First, PAI-1 was successfully immobilized at $10 \mu \mathrm{g} / \mathrm{mL}$ (the same concentration used for immobilization of other bioreceptors, i.e. scFv-33H1F7 or MDTCS) in TBS/Tween directly on the FO-NTA probe with a final shift of about $6.5 \mathrm{~nm}$ (Figure S3A). Second, when performing the sandwich bioassay for PAI-1 detection (400 $\mathrm{ng} / \mathrm{mL}$ ) in the absence of any bioreceptors on the FO probes, no signal amplification was achieved by AuNPs functionalized with MA-31C9, contrary to the sandwich bioassays performed with scFv-33H1F7 bioreceptors on the FO probes (see further below). This is because the $\mathrm{His}^{3}$ residue of PAI-1 (labelled in Figure S3B) that causes the non-specific binding of PAI-1 to the NTA surface also contributes to the epitope of MA-31C9 as previously reported (Figure S3C). ${ }^{52}$ Third, Figure S3D and Figure S3E demonstrated the insufficient oxidation from $\mathrm{Co}^{2+}$ to $\mathrm{Co}^{3+}$ when the Co(II)-NTA surface was not functionalized with scFv-33H1F7 or PAI-1. The above-obtained data further emphasized the importance of saturating the FO probe with specific His 6 -tagged bioreceptors in order to prevent non-specific interaction of PAI-1 with the NTA surface. However, for knowing the minimum amount of bioreceptors on the FO probes to achieve this, we immobilized different concentrations of MDTCS bioreceptors $(10,5,2.5,1.25$ and $0.625 \mu \mathrm{g} / \mathrm{mL})$ as depicted in Figure S4. MDTCS bioreceptor was selected for this experiment based on the observed absence of PAI-1 
interaction with MDTCS (when MDTCS is immobilized at $10 \mu \mathrm{g} / \mathrm{mL}$, Figure 3), which therefore allows us to discern if the non-specific binding of PAI-1 occurs directly to NTA SAM. According to the results in Figure 4B, PAI-1 nonspecific binding against scFv-33H1F7 was steadily increasing with the decreasing concentration of MDTCS bioreceptors on the FO probe surface, further proving that PAI-1 can indeed directly interact with the NTA SAM when the surface is not saturated with the bioreceptors. Moreover, $5 \mu \mathrm{g} / \mathrm{mL}$ of MDTCS seemed to be already a critical point for observing slightly higher non-specific binding compared to the one obtained for $10 \mu \mathrm{g} / \mathrm{mL}$ of MDTCS, which was therefore taken into account in the subsequent experiments.
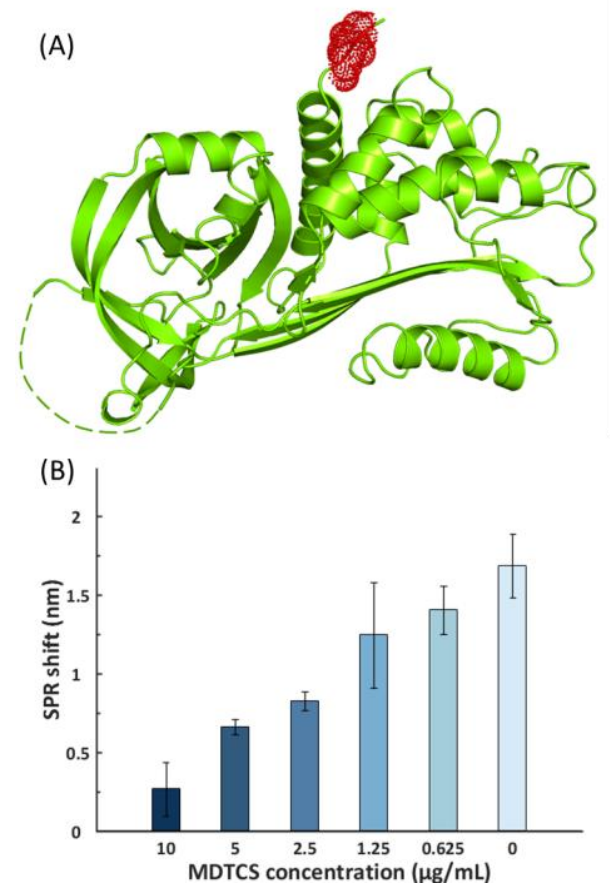

Figure 4. (A) Cartoon representation of the crystal structure of PAI-1, with two consecutive histidines exposed on the surface (highlighted in red). This figure was generated using the PDB file (ID: 6GWN) with PyMOL software (Molecular Graphics System, Version 2.0.7, Schrödinger, LLC). ${ }^{53}$ (B) SPR shifts of PAI-1 binding to the FO probe surface in label-free bioassay when MDTCS is immobilized at different concentrations ( $0-10 \mu \mathrm{g} / \mathrm{mL})$. Error bars represent standard deviations $\left(\mathrm{n}_{\mathrm{s}}=2\right)$.

\section{Establishing label-free bioassay in buffer}

To further explore the potential of Co(III)-NTA chemistry, we first investigated the impact that different immobilization kinetics and the number of bioreceptors might have on the FO-SPR sensing performance in the buffer, including the sensitivity and reproducibility of the established bioassay. Therefore, we immobilized scFv33H1F7 bioreceptors on the FO probes using different concentrations $(20,10$, and $5 \mu \mathrm{g} / \mathrm{mL})$ and performed labelfree detection of PAI-1. Importantly, to protect the unsaturated FO probes from non-specific binding of PAI-1 when using only $5 \mu \mathrm{g} / \mathrm{mL}$ of scFv-33H1F7 (Figure 2), we added $5 \mu \mathrm{g} / \mathrm{mL}$ of MDTCS to formulate a mixture of His6tagged bioreceptors at the final concentration of $10 \mu \mathrm{g} / \mathrm{mL}$. This was proven feasible without affecting the interaction between scFv-33H1F7 and PAI-1 as indicated by Figure S5.

Calibration curves were first established for all three scFv33H1F7 concentrations in the buffer and fitted throughout the entire measured PAI-1 range with a non-linear regression for $30 \mathrm{~min}$ of the bioassay (i.e. $30 \mathrm{~min}$ of the interaction between PAI-1 and scFv-33H1F7, Figure 5). The calculated LOD estimates at 95\% confidence intervals are displayed in Table 1. As expected, the calibration curves generated with 20 and $10 \mu \mathrm{g} / \mathrm{mL}$ of scFv-33H1F7 were greatly overlapping throughout the entire range of tested PAI-1 concentrations, resulting also in comparable LOD values (from t-test, as shown in Table S1), again emphasizing that the latter concentration of bioreceptors is adequate and sufficient when using NTA surface chemistry. Surprisingly, however, the calculated p-values of more than 0.05 (Table S1) indicated that there was no significant difference among obtained LOD values even when only 5 $\mu \mathrm{g} / \mathrm{mL}$ of scFv-33H1F7 was immobilized, although the SPR shifts for the highest three PAI-1 concentrations were substantially lower compared to those obtained when using 10 or $20 \mu \mathrm{g} / \mathrm{mL}$ of scFv-33H1F7. This suggested that the observed $18 \%$ difference in immobilized scFv-33H1F7 bioreceptors on the FO probe surface (Figure 2A) did not impact the sensitivity of the label-free bioassays in the buffer, probably owing to the oriented patterning of bioreceptors. The obtained average coefficients of variation (CVs) being $7 \%, 2 \%$ and $4 \%$ for 20,10 , and $5 \mu \mathrm{g} / \mathrm{mL}$ of scFv-33H1F7, respectively, suggested a very high reproducibility of the implemented bioassay.

Interestingly, the sensitivity of the FO probes with the NTA surface was significantly improved in label-free bioassays compared to the FO probes with the $\mathrm{COOH}$ surface. For instance, our previously developed $\mathrm{COOH}$-based label-free bioassay for detecting $10 \mu \mathrm{g} / \mathrm{mL}$ of infliximab (IFX) resulted in approximately $1 \mathrm{~nm}$ SPR shift when using the optimized FO probe functionalized with $20 \mu \mathrm{g} / \mathrm{mL}$ of antibody. ${ }^{6}$ Contrary to this, functionalized FO probes with either 20 or $10 \mu \mathrm{g} / \mathrm{mL}$ of scFv-33H1F7 gave about $6.5 \mathrm{~nm}$ SPR shift in $30 \mathrm{~min}$ of the label-free bioassay when detecting $8 \mu \mathrm{g} / \mathrm{mL}$ of PAI-1 (Figure 4 ). This suggests that oriented immobilization of bioreceptors on the sensor surface might be one of the important factors towards the successful implementation of label-free bioassays on the FO-SPR platform. In this context it is important to note that, although the difference in size between NTA and $\mathrm{COOH}$ SAM can be neglected, the difference in size between scFv33H1F7 and IgG antibody might be another significant factor that contributed to achieving a higher detection signal shift (due to the shorter distance between gold surface and the available binding sites for the target molecules). Although just a hypothesis here, this can be a subject of further study to understand to what extent the size of bioreceptors might play a role in the establishment of label-free bioassays, next to their better orientation. Moreover, the final comparison of two surface chemistries 
will require several model systems to test whether the

observed differences are bioassay independent.

Table 1. Calculated LOD estimates at $95 \%$ confidence intervals for different bioassay types at various conditions. The mean values were calculated by averaging the minimum and maximum values of the estimated range.

\begin{tabular}{|c|c|c|c|c|c|c|c|}
\hline \multirow{2}{*}{\multicolumn{2}{|c|}{$\begin{array}{l}\text { ScFv-33H1F7 concentration } \\
\text { Bioassay type } \\
\text { Label-free bioassay } \\
\text { (LOD expressed in } \mu \mathrm{g} / \mathrm{mL} \text { ) }\end{array}$}} & \multicolumn{2}{|c|}{$20(\mu \mathrm{g} / \mathrm{mL})$} & \multicolumn{2}{|c|}{$10(\mu \mathrm{g} / \mathrm{mL})$} & \multicolumn{2}{|c|}{$5(\mu \mathrm{g} / \mathrm{mL})$} \\
\hline & & Range & Mean & Range & Mean & Range & Mean \\
\hline $\begin{array}{l}\text { Buffer } \\
\text { 20x pla }\end{array}$ & sma & $\begin{array}{c}0.06-0.64 \\
/\end{array}$ & $\begin{array}{c}0.35 \\
/\end{array}$ & $\begin{array}{l}0.29-0.86 \\
0.05-1.11\end{array}$ & $\begin{array}{l}0.58 \\
0.58\end{array}$ & $\begin{array}{c}0.01-0.59 \\
/\end{array}$ & $\begin{array}{c}0.30 \\
/\end{array}$ \\
\hline $\begin{array}{l}\text { Sandwi } \\
\text { (LOD ex }\end{array}$ & $\begin{array}{l}\text { ch bioassay } \\
\text { pressed in } \mathrm{ng} / \mathrm{mL} \text { ) }\end{array}$ & Range & Mean & Range & Mean & Range & Mean \\
\hline $\begin{array}{l}\text { Buffer } \\
10 x \text { pla }\end{array}$ & sma & $\begin{array}{c}5.27-20.37 \\
/\end{array}$ & $\begin{array}{c}12.82 \\
/\end{array}$ & $\begin{array}{l}4.81-10.83 \\
3.85-11.69\end{array}$ & $\begin{array}{l}7.82 \\
7.77\end{array}$ & $\begin{array}{c}-0.14-12.66 \\
/\end{array}$ & $\begin{array}{c}6.26 \\
/\end{array}$ \\
\hline$\underbrace{7}$ & $\begin{array}{l}\mathrm{y}=\mathrm{A}^{*} \mathrm{x} /(\mathrm{B}+\mathrm{x}) \\
\mathrm{I:} \text { Adj. } \mathrm{R}^{2}=0.976 \\
\text { II: Adj. } \mathrm{R}^{2}=0.991 \\
\text { III: Adj. } \mathrm{R}^{2}=0.971\end{array}$ & 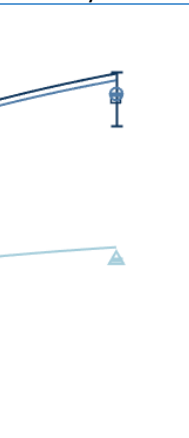 & \multicolumn{5}{|c|}{$\begin{array}{l}\text { The acquired average SPR shifts }(\mathrm{n}=4) \text { in } 30 \text { min were } \\
\text { plotted as a function of the PAI- } 1 \text { concentration and fitted } \\
\text { through non-linear regression (Figure 6), resulting in an } \\
\text { average CV value of } 13 \% \text { and calculated LOD estimates as } \\
\text { depicted in Table } 1 \text {. Compared to the label-free bioassay of } \\
\text { PAI-1 in the buffer, at either scFv-33H1F7 concentration } \\
\text { there was no significant difference among LOD estimates } \\
\text { based on the calculated p-values from the t-test (Table S1) } \\
\text { Therefore, the sensitivity of the label-free bioassay was not } \\
\text { affected when applied in diluted plasma, validating the } \\
\text { surface stability in a complex matrix. }\end{array}$} \\
\hline
\end{tabular}

Expanding the dynamic detection range through AuNPsmediated signal amplification

Many biomarkers, including PAI-1, need to be detected in clinical samples at concentrations lower than the nanogram/mL (e.g. 0-50 ng/mL is clinically relevant range for PAI-1).54 Therefore, we wanted to test whether signal amplification strategy with AuNPs (functionalized with MA-31C9) can be implemented on the Co(III)-NTA surface to increase bioassay sensitivity in a similar manner as previously described for the $\mathrm{COOH}$-based surface chemistry on the FO-SPR platform. ${ }^{6}$ Therefore, a series of much lower PAI-1 concentrations than those detected with the label-free bioassay, ranging from 0 to $400 \mathrm{ng} / \mathrm{mL}$, was spiked and detected in a sandwich bioassay both in the buffer and diluted plasma. The obtained SPR shifts were averaged and plotted as a function of the PAI-1 concentration to generate calibration curves for 20,10 and $5 \mu \mathrm{g} / \mathrm{mL}$ of scFv-33H1F7 bioreceptor in buffer (Figure 7A) and for $10 \mu \mathrm{g} / \mathrm{mL}$ of scFv-33H1F7 in diluted plasma (Figure 7B), resulting in the LOD estimates as depicted in Table 1. , we first tested 10,20, 50 and 100-fold dilute plasma samples without spiked PAI-1. As shown in Figure S6A, non-specific binding decreased starting from 20 -fold dilution onwards with, moreover, similar values observed for 20, 50 and 100-fold dilutions. Therefore, 20-fold diluted plasma was deemed as the most appropriate for spiking with PAI-1 (0 to $8 \mu \mathrm{g} / \mathrm{mL})$ in order to generate the calibration curve, with obtained minimal and maximal signal as shown in Figure S6B. To ensure high sensitivity and avoid non-specific binding resulting from an unsaturated sensor surface, as explained above, $10 \mu \mathrm{g} / \mathrm{mL}$ of scFv-33H1F7 was immobilized on the F0 probe surface. 


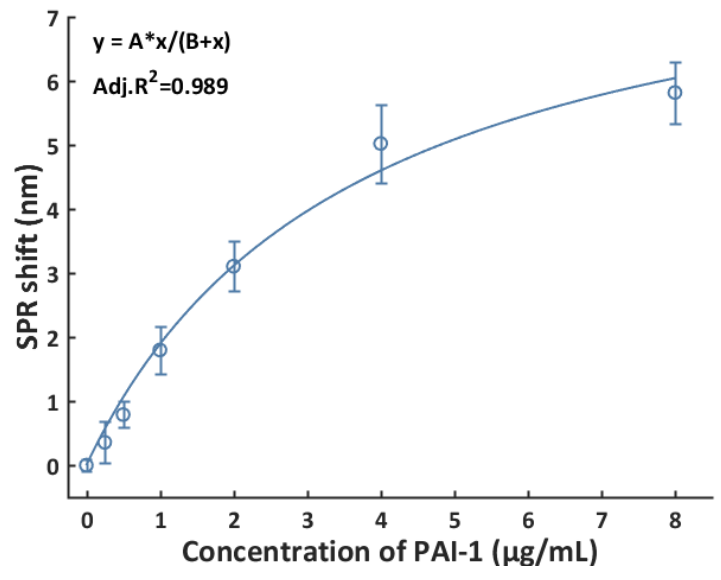

Figure 6. Calibration curve obtained from a series of PAI-1 concentrations in 20-fold diluted plasma for label-free bioassay, with non-linear (one-site binding) regression fitting. Fitting parameters are as indicated here $(A=8.81 \mathrm{~nm}, \mathrm{~B}=3.66$ $\mu \mathrm{g} / \mathrm{mL}, \mathrm{RMSE}=0.23 \mu \mathrm{g} / \mathrm{mL}$ ). Error bars represent standard deviations $\left(\mathrm{n}_{\mathrm{s}}=4\right)$.

When performed in the buffer, the sandwich bioassay resulted in average $\mathrm{CV}$ values of $6 \%, 5 \%$ and $5 \%$ for 20,10 and $5 \mu \mathrm{g} / \mathrm{mL}$ of scFv-33H1F7, respectively, again emphasizing high reproducibility. Moreover, the obtained LOD estimates of these three bioassays indicated the possibility to successfully combine AuNPs-mediated SPR signal enhancements with the Co(III)-NTA surface, bringing down the target detection range from $\mu \mathrm{g} / \mathrm{mL}$ to low ng/mL. Based on the calculated $\mathrm{p}$-values in the t-test (Table S1), it seemed that there was no significant difference among the LOD estimates obtained when immobilizing three different concentrations of scFv33H1F7 bioreceptors. Collectively, these results suggested that the reproducibility and sensitivity of the developed sandwich bioassays in the buffer, as well as their accuracy (based on the $\mathrm{R}^{2}$ and RMSE values), were not significantly affected by a lower concentration of bioreceptors on the FO probe surface. In line with this, the calibration curves obtained with three different scFv-33H1F7 concentrations largely overlapped, especially for the lowest PAI-1 concentrations. Interestingly, the discrepancy in a signal shift for the highest measured PAI-1 concentration between $5 \mu \mathrm{g} / \mathrm{mL}$ of scFv-33H1F7 and 20 or $10 \mu \mathrm{g} / \mathrm{mL}$ was only $20 \%$ for the sandwich bioassay, whereas, in the labelfree bioassay, the observed difference was $42 \%$ when comparing the same conditions. The smaller discrepancy for the sandwich bioassay could be due to the diffusion limitations of AuNPs and/or saturation of the FO-SPR sensor surface with AuNPs, thereby leaving some of the bioreceptors at higher scFv-33H1F7 concentrations effectively inaccessible for binding even more AuNPs. Although outside the scope of this paper, it would be of interest to further understand whether (1) introduced shaking during the AuNPs binding step, (2) smaller-sized AuNPs or (3) combination of both could further improve the overall bioassay sensitivity by counteracting the diffusion limitation and/or allowing engagement of more bioreceptors on the surface.
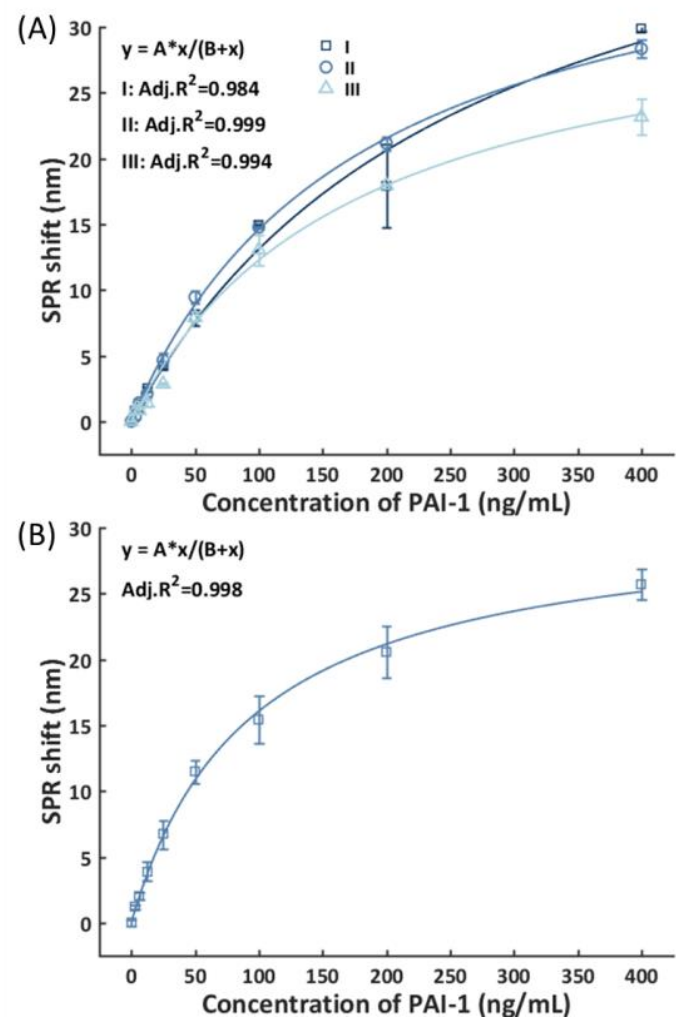

Figure 7. Summary of calibration curves for sandwich bioassay obtained from series of PAI-1 concentrations in buffer (A) and 10-fold diluted plasma (B). Non-linear regression was used to fit all calibration curves. (A) scFv33H1F7 was immobilized at 20 (I), 10 (II) and 5 (III) $\mu \mathrm{g} / \mathrm{mL}$. Fitting parameters are as indicated here (I: $A=48.24 \mathrm{~nm}$, $\mathrm{B}=268.22 \mathrm{ng} / \mathrm{mL}, \quad \mathrm{RMSE}=1.15 \mathrm{ng} / \mathrm{mL} ; \quad \mathrm{II}: \mathrm{A}=40.96 \mathrm{~nm}$, $\mathrm{B}=180.54 \mathrm{ng} / \mathrm{mL}, \quad \mathrm{RMSE}=0.34 \mathrm{ng} / \mathrm{mL} ; \mathrm{III}: \mathrm{A}=33.49 \mathrm{~nm}$, $\mathrm{B}=173.10 \mathrm{ng} / \mathrm{mL}$, RMSE $=0.63 \mathrm{ng} / \mathrm{mL}$ ). Error bars represent standard deviations $\left(\mathrm{n}_{\mathrm{s}}=2\right)$. (B) scFv-33H1F7 was immobilized at $10 \mu \mathrm{g} / \mathrm{mL}$. Fitting parameters are $A=30.98 \mathrm{~nm}$, $\mathrm{B}=92.88 \mathrm{ng} / \mathrm{mL}, \mathrm{RMSE}=0.42 \mathrm{ng} / \mathrm{mL}$. Error bars represent standard deviations $\left(\mathrm{n}_{\mathrm{s}}=4\right)$.

Furthermore, to explore the applicability and sensitivity of the developed sandwich bioassay in complex matrices, measurement of PAI-1 was performed in 10-fold diluted plasma with spiked PAI-1 ranging from $0-400 \mathrm{ng} / \mathrm{mL} .10-$ fold dilution was used here to demonstrate that minimal dilution of complex matrices is attainable when performing the sandwich bioassay with the NTA surface chemistry, similarly to our previous work where sandwich bioassays were developed using $\mathrm{COOH}$ SAM for detecting targets in 10 -fold diluted whole blood ${ }^{1,6}$. With an average CV of $8 \%$ and no significant difference observed among the calculated LOD estimates in plasma samples and those in buffer at each scFv-33H1F7 concentration (Table 1 and Table S1), we can conclude that the complex matrix did not impact the sensitivity or accuracy of the sandwich bioassay. This is in contrast to our previous study with $\mathrm{COOH}$ SAM on the FO probes, where approximately 10 times higher LOD values were obtained for the sandwich bioassay in 100 -fold diluted serum than in buffer. ${ }^{6}$ Although further work is needed to test whether these results are not 
bioassay dependent, it seems that NTA surface chemistry can endure the impact resulted from complex matrices at least as well if not even better than $\mathrm{COOH}$ based one.

\section{CONCLUSIONS}

In the current study, we have applied Co(III)-NTA chemistry to realize oriented and stable patterning of His6tagged bioreceptors on the FO-SPR platform for the first time. Firstly, we tested the efficiency of immobilizing His6tagged scFv-33H1F7 bioreceptors at different concentrations, i.e. 20,10 and $5 \mu \mathrm{g} / \mathrm{mL}$ on the NTA surface. 20 and $10 \mu \mathrm{g} / \mathrm{mL}$ of scFv-33H1F7 resulted in comparable number of bioreceptors immobilized on the surface with only noticeable difference in kinetics. Interestingly, decreasing the scFv-33H1F7 concentration to $5 \mu \mathrm{g} / \mathrm{mL}$ resulted in a final immobilization shift that was $80 \%$ of the signal obtained for 2- or 4-fold higher scFv-33H1F7 concentrations, with evidently slower immobilization kinetics. Compared to the previous case studies using $\mathrm{COOH}$ SAM in our group, the acquired SPR shifts for immobilization were significantly higher (i.e. $>3 \mathrm{~nm}$ ) using NTA SAM, suggesting that the oriented patterning of bioreceptors mediated by NTA SAM might have a role in more efficient surface coverage, thereby resulting in more bioreceptors immobilized on the sensor surface. This difference is even more pronounced when taking into account that the bioreceptor in this study is an antibody fragment, unlike previously used full-size IgG. Moreover, contrary to the previously optimized $20 \mu \mathrm{g} / \mathrm{mL}$ of bioreceptors to be used on the $\mathrm{COOH}$ SAM, $10 \mu \mathrm{g} / \mathrm{mL}$ of scFv-33H1F7 was considered as sufficient to saturate the NTA surface and ensured a good sensitivity.

Since PAI-1 was selected as a model system, a series of control tests were performed to prove the specific binding between PAI-1 and scFv-33H1F7. Although non-specific binding occurred between PAI-1 and unsaturated NTA surface due to the two consecutive histidines in the PAI-1 sequence, $5 \mu \mathrm{g} / \mathrm{mL}$ of another His ${ }^{-}$-tagged bioreceptors (MDTCS) was co-immobilized with $5 \mu \mathrm{g} / \mathrm{mL}$ of scFv33H1F7 to cover the unsaturated surface, thereby preventing the non-specific binding of PAI-1.

Secondly, we applied Co(III)-NTA strategy to develop labelfree bioassays of PAI-1 both in a buffer (with 20, 10 or 5 $\mu \mathrm{g} / \mathrm{mL}$ of $\mathrm{scFv}-33 \mathrm{H} 1 \mathrm{~F} 7$ ) and in 20-fold diluted plasma using $10 \mu \mathrm{g} / \mathrm{mL}$ of scFv-33H1F7 immobilized on the F0 probes. Calibration curves were made with averaged SPR signal shifts as a function of PAI-1 concentrations, ranging from 0 to $8 \mu \mathrm{g} / \mathrm{mL}$. LOD estimates were calculated, taking into account standard deviations in both blank and test samples, the fitting equation as well as a $95 \%$ confidence interval, in order to statistically compare the sensitivity of different bioassays by t-test. Collectively, both the accuracy and sensitivity of established label-free bioassay were comparable at different number of immobilized bioreceptors, as well as among buffer and plasma samples. When rivaled to the earlier reported label-free bioassays built on the $\mathrm{COOH}$ FO-SPR surface, there was an apparent NTA-mediated increase in bioassay sensitivity. Although this has been shown with only one model system here and would have to be further validated with additional cases, it certainly displays a fresh potential of the FO-SPR platform for so far difficult-to-attain label-free bioassays. Moreover, a considerable reduction in the bioreceptor number on the surface without affecting the overall sensitivity, might also offer a great prospect in building a multiplex bioassay on the FO-SPR sensor as it opens up the opportunity to functionalize the surface with multiple bioreceptors at low concentration before reaching its full saturation.

Finally, due to the nanomolar detecting requirements in clinical samples for many biomarkers, including PAI-1, a sandwich bioassay was established using AuNPs-mediated signal amplification. Equivalent to the label-free bioassay, different scFv-33H1F7 concentrations did not affect the sensitivity or accuracy of the established sandwich bioassay. Notably, the same sensitivity endured even when the bioassay was performed in 10-fold diluted plasma, revealing that the complexity of the matrix had no pronounced effect on the NTA-based SPR surface.

Overall, this study demonstrated for the first time the feasibility of oriented and stable patterning of bioreceptors using Co(III)-NTA strategy on the FO-SPR sensor surface. This contributed to having surfaces more tolerant towards the different number of immobilized bioreceptors next to showing necessary stability for developing sensitive bioassays in complex matrices, such as plasma.

\section{ASSOCIATED CONTENT}

\section{Supporting information}

The Supporting Information is available free of charge.

The Supporting information file contains: Image of the FOSPR platform from FOx Biosystems, sensorgram depicting functionalization of the FO probe, detailed explanation about the non-specific binding between PAI-1 and the NTA surface including the amino acid sequence of PAI-1, extra experimental results regarding MDTCS immobilization on the FO probe, selection of plasma dilution factors, p-values from the t-test, and references (PDF).

\section{AUTHOR INFORMATION}

\section{Corresponding author}

*E-mail: jeroen.lammertyn@kuleuven.be

ORCID

Jeroen Lammertyn: 0000-0001-8143-6794

\section{Author Contributions}

J.H. Qu designed and optimized the protocols, carried out experiments, analyzed data and wrote the manuscript; S.H. was involved in discussions and provided technical help in the lab; F.D. contributed to the interpretation of the results and provided technical help in the lab; N.G, P.D, M.S. and K.V. provided biological reagents and were involved in discussions; D.-W. S. was involved in discussions and revised the manuscript. J.L. conceived the study, was in charge of overall 
direction and planning and prepared the manuscript; D.S. designed the experiments, supervised work, provided discussions and wrote the manuscript. All authors discussed the results and provided their critical feedback on the manuscript. All authors have given approval to the final version of the manuscript.

\section{ACKNOWLEDGMENTS}

This work has received funding from Guangzhou Elite Project, the European Union's Horizon 2020 research and innovation program under the Marie Skłodowska-Curie grant (agreement no. 675412, H2020-MSCA-ITN-ND4ID), the European Union's Horizon 2020 research and innovation program under the Marie Skłodowska-Curie grant (agreement no.675746, H2020MSCA-ITN-PROFILE) and the Fonds Wetenschappelijk Onderzoek-Toegepast Biomedisch Onderzoek met een primair Maatschappelijke finaliteit (FWO-TBM grant no T002918N). M. $\mathrm{S}$. is a recipient of a $\mathrm{Ph}$. D. fellowship of the Research Foundation - Flanders (FWO). We would like to thank Prof. Jan Voorberg (Department of Plasma Proteins, SanquinAcademic Medical Center Landsteiner Laboratory, Amsterdam, the Netherlands) for providing monoclonal antibody II-1.

\section{REFERENCES}

(1) Lu, J.; Spasic, D.; Delport, F.; Van Stappen, T.; Detrez, I.; Daems, D.; Vermeire, V.; Gils, A.; Lammertyn, J.; Leuven, K. Immunoassay for Detection of Infliximab in Whole Blood Using a Fiber-Optic Surface Plasmon Resonance Biosensor. Anal. Chem. 2017, 89, 3664-3671.

(2) Ravalli, A.; da Rocha, C. G.; Yamanaka, H.; Marrazza, G. A Label-Free Electrochemical Affisensor for Cancer Marker Detection: The Case of HER2. Bioelectrochemistry 2015, 106, 268-275.

(3) Pollet, J.; Delport, F.; Janssen, K. P. F.; Jans, K.; Maes, G.; Pfeiffer, H.; Wevers, M.; Lammertyn, J. Fiber Optic SPR Biosensing of DNA Hybridization and DNA-Protein Interactions. Biosens. Bioelectron. 2009, 25, 864-869.

(4) Kugimiya, A.; Takeuchi, T. Surface Plasmon Resonance Sensor Using Molecularly Imprinted Polymer for Detection of Sialic Acid. Biosens. Bioelectron. 2001, 16, 1059-1062.

(5) Grieshaber, D.; MacKenzie, R.; Vörös, J.; Reimhult, E. Electrochemical Biosensors - Sensor Principles and Architectures. Sensors 2008, 8, 1400-1458.

(6) Lu, J.; Van Stappen, T.; Spasic, D.; Delport, F.; Vermeire, S.; Gils, A.; Lammertyn, J.; Leuven, K. Fiber Optic-SPR Platform for Fast and Sensitive Infliximab Detection in Serum of Inflammatory Bowel Disease Patients. Biosens. Bioelectron. 2017, 79, 173-179.

(7) Kausaite-Minkstimiene, A.; Ramanaviciene, A.; Kirlyte, J.; Ramanavicius, A. Comparative Study of Random and Oriented Antibody Immobilization Techniques on the Binding Capacity of Immunosensor. Anal. Chem. 2010, 82, 6401-6408.

(8) Hodneland, C. D.; Lee, Y.-S.; Min, D.-H.; Mrksich, M. Selective Immobilization of Proteins to Self-Assembled Monolayers Presenting Active Site-Directed Capture Ligands. Proc. Natl. Acad. Sci. 2002, 99, 5048-5052.

(9) Khan, F.; He, M.; Taussig, M. J. Double-Hexahistidine Tag with HighAffinity Binding for Protein Immobilization, Purification, and Detection on Ni-Nitrilotriacetic Acid Surfaces. Anal. Chem. 2006, 78, 3072-3079.

(10) Gautrot, J. E.; Huck, W. T. S.; Welch, M.; Ramstedt, M. ProteinResistant NTA-Functionalized Polymer Brushes for Selective and Stable Immobilization of Histidine-Tagged Proteins. ACS Appl. Mater. Interfaces 2010, 2, 193-202.

(11) Shen, W.; Zhong, H.; Neff, D.; Norton, M. L. NTA Directed Protein Nanopatterning on DNA Origami Nanoconstructs. J. AM. CHEM. SOC 2009, 131, 6660-6661.

(12) Hochuli, E.; Bannwarth, W.; Döbeli, H.; Gentz, R.; Stüber, D. Genetic Approach to Facilitate Purification of Recombinant Proteins with a Novel Metal Chelate Adsorbent. Nat. Biotechnol. 1988, 6, 1321-1325.

(13) Mourão, C. A.; Carmignotto, G. P.; Bueno, S. M. A. Separation of
Human IgG Fragments Using Copper, Nickel, Zinc, and Cobalt Chelated to CM-Asp-Agarose by Positive and Negative Chromatography. J. Chromatogr. B Anal. Technol. Biomed. Life Sci. 2016, 1017-1018, 163-173.

(14) Chaga, G.; Hopp, J.; Nelson, P. Immobilized Metal Ion Affinity Chromatography on Co2+-carboxymethylaspartate-Agarose Superflow, as Demonstrated by One-step Purification of Lactate. Biotechnol. Appl. 1999, 24, 19-24.

(15) Gaberc-porekar, V.; Menart, V. Perspectives of Immobilized-Metal Naffinity Chromatography. J. Biochem. Biophys. Methods 2001, 49, 335-360.

(16) Gout, P. W.; Morganti, L. Current and Prospective Applications of Metal Ion-Protein Binding. 2003, 988, 1-23.

(17) Porath, J. Immobilized Metal Ion Affinity Chromatography. Protein Expr. Purif. 1992, 3, 263-281.

(18) Porath, J.; Carlsson, J.; Olsson, I.; Belfrage, G. Metal Chelate Affinity Chromatography, a New Approach to Protein Fractionation. Nature 1975, 258, 598-599.

(19) Lippard, S. J.; Berg, J. M. Principles of Bioinorganic Chemistry; University Science Books, 1994.

(20) Rodgers, M. A.; Findlay, J. B. C.; Millner, P. A. Lipocalin Based Biosensors for Low Mass Hydrophobic Analytes; Development of a Novel SAM for Polyhistidine Tagged Proteins. Sensors Actuators, B Chem. 2010, 150, 12-18.

(21) Zhang, H.; Li, Z. fei; Snyder, A.; Xie, J.; Stanciu, L. A. Functionalized Graphene Oxide for the Fabrication of Paraoxon Biosensors. Anal. Chim. Acta 2014, 827, 86-94.

(22) Li, X.; Song, S.; Pei, Y.; Dong, H.; Aastrup, T.; Pei, Z. Oriented and Reversible Immobilization of His-Tagged Proteins on Two- and Three-Dimensional Surfaces for Study of Protein-Protein Interactions by a QCM Biosensor. Sensors Actuators, B Chem. 2016, 224, 814-822.

(23) Clow, F.; Fraser, J. D.; Proft, T. Immobilization of Proteins to Biacore Sensor Chips Using Staphylococcus Aureus Sortase A. Biotechnol. Lett. 2008, 30, 1603-1607.

(24) Willard, F. S.; Siderovski, D. P. Covalent Immobilization of Histidine-Tagged Proteins for Surface Plasmon Resonance. Anal. Biochem. 2006, 353, 147-149.

(25) Kimple, A. J.; Muller, R. E.; Siderovski, D. P.; Willard, F. S. A Capture Coupling Method for the Covalent Immobilization of Hexahistidine Tagged Proteins for Surface Plasmon Resonance. In Surface Plasmon Resonance; Springer, 2010; pp 91-100.

(26) Wegner, S. V.; Spatz, J. P. Cobalt(III) as a Stable and Inert Mediator Ion between NTA and His6-Tagged Proteins. Angew. Chemie - Int. Ed. 2013, 52, 7593-7596.

(27) Valiokas, R.; Klenkar, G.; Tinazli, A.; Tampé, R.; Liedberg, B.; Piehler, J. Differential Protein Assembly on Micropatterned Surfaces with Tailored Molecular and Surface Multivalency. ChemBioChem 2006, 7, 1325-1329.

(28) Valiokas, R.; Klenkar, G.; Tinazli, A.; Reichel, A.; Tampé, R.; Piehler, J.; Liedberg, B. Self-Assembled Monolayers Containing Terminal Mono-, Bis-, and Tris-Nitrilotriacetic Acid Groups: Characterization and Application. Langmuir 2008, 24, 4959-4967.

(29) Lata, S.; Reichel, A.; Brock, R.; Tampé, R.; Piehler, J. High-Affinity Adaptors for Switchable Recognition of Histidine-Tagged Proteins. J. Am. Chem. Soc. 2005, 127, 10205-10215.

(30) Wang, X.; Liu, Q.; Tan, X.; Liu, L.; Zhou, F. Covalent Affixation of Histidine-Tagged Proteins Tethered onto Ni-Nitrilotriacetic Acid Sensors for Enhanced Surface Plasmon Resonance Detection of Small Molecule Drugs and Kinetic Studies of Antibody/Antigen Interactions. Analyst 2019, 144, 587-593.

(31) Wegner, S. V.; Schenk, F. C.; Witzel, S.; Bialas, F.; Spatz, J. P. Cobalt Cross-Linked Redox-Responsive PEG Hydrogels: From Viscoelastic Liquids to Elastic Solids. Macromolecules 2016, 49, 4229-4235.

(32) Wegner, S. V.; Schenk, F. C.; Spatz, J. P. Cobalt(III)-Mediated Permanent and Stable Immobilization of Histidine-Tagged Proteins on NTA-Functionalized Surfaces. Chem. - A Eur. J. 2016, 22, 31563162.

(33) Visser, H. G.; Purcell, W.; Basson, S. S. Kinetic Study of the Protonations and Substitutions of Different Cobalt(III)-Nta Complexes. Transit. Met. Chem. 2002, 27, 461-468.

(34) Ogino, H.; Ogino, K. Redox Potentials and Related Parameters of 
Cobalt (III/II) Complexes Containing Aminopolycarboxylates. Inorg Chem. 1983, 22, 2208-2211.

(35) Bond, B. J.; Hobson, B. The Stability Constants of Cobalt(Iii) Chelates of Polyaminopolycarboxylic Acids. J. Chem. Soc. A Inorganic, Phys. Theor. 1969, 2155-2157.

(36) Auer, S.; Azizi, L.; Faschinger, F.; Blazevic, V.; Vesikari, T.; Gruber, H. J.; Hytönen, V. P. Stable Immobilisation of His-Tagged Proteins on BLI Biosensor Surface Using Cobalt. Sensors Actuators B Chem. 2017, 243, 104-113.

(37) Shao, S.; Geng, J.; Yi, H. A.; Gogia, S.; Neelamegham, S.; Jacobs, A.; Lovell, J. F. Functionalization of Cobalt Porphyrin-Phospholipid Bilayers with His-Tagged Ligands and Antigens. Nat. Chem. 2015, 7 , 438-446.

(38) Bijnens, A.-P.; Gils, A.; Knockaert, I.; Stassen, J. M.; Declerck, P. J. Importance of the Hinge Region Between-Helix F and the Main Part of Serpins, Based upon Identification of the Epitope of Plasminogen Activator Inhibitor Type 1 Neutralizing Antibodies*. J. Biol. Chem 2000, 275 (9), 6375-6380.

(39) Roca, C.; Primo, L.; Valdembri, D.; Cividalli, A.; Declerck, P. Carmeliet, P.; Gabriele, P.; Bussolino, F. Hyperthermia Inhibits Angiogenesis by a Plasminogen Activator Inhibitor 1-Dependent Mechanism 1. Cancer Res. 2003, 63, 1500-1507.

(40) Declerck, P. J.; Alessi, M.-C.; Verstreken, M.; Kruithof, E. K. O.; Irene, J.-V.; Collen, D. Measurement of Plasminogen Activator Inhibitor 1 in Biologic Fluids with a Murine Monoclonal Antibody-Based EnzymeLinked Immunosorbent Assay. Blood 1988, 71, 220-225.

(41) Kremer Hovinga, J. A.; Coppo, P.; Lämmle, B.; Moake, J. L.; Miyata, T.; Vanhoorelbeke, K. Thrombotic Thrombocytopenic Purpura. Nat. Rev. Dis. Prim. 2017, 3 (1), 17020.

(42) Deforche, L.; Roose, E.; Vandenbulcke, A.; Vandeputte, N.; Feys, H. B.; Springer, T. A.; Mi, L. Z.; Muia, J.; Sadler, J. E.; Soejima, K.; et al. Linker Regions and Flexibility around the Metalloprotease Domain Account for Conformational Activation of ADAMTS-13. J. Thromb. Haemost. 2015, 13 (11), 2063-2075.

(43) POS, W.; LUKEN, B. M.; KREMER HOVINGA, J. A.; TURENHOUT, E. A M.; SCHEIFLINGER, F.; DONG, J.-F.; FIJNHEER, R.; VOORBERG, J. VH1 69 Germline Encoded Antibodies Directed towards ADAMTS13 in Patients with Acquired Thrombotic Thrombocytopenic Purpura. J. Thromb. Haemost. 2009, 7 (3), 421-428.

(44) Shimba, N., Takahashi, H., Sakakura, M., Fujii, I., Shimada, I. Determination of Protonation and Deprotonation Forms and
Tautomeric States of Histidine Residues in Large Proteins Using Nitrogen- Carbon J Couplings in Imidazole Ring. J. Am. Chem. Soc. 1998, 120, 10988-10989.

(45) Arghir, I.; Spasic, D.; Verlinden, B. E.; Delport, F.; Lammertyn, J. Improved Surface Plasmon Resonance Biosensing Using Silanized Optical Fibers. Sensors Actuators B Chem. 2015, 216, 518-526.

(46) Holstein, C. A.; Griffin, M.; Hong, J.; Sampson, P. D. Statistical Method for Determining and Comparing Limits of Detection of Bioassays. Anal. Chem. 2015, 87, 9795-9801.

(47) Pollet, J. Fiber Optic Surface Plasmon Resonance, KU Leuven, 2010.

(48) Royhaila Mohamad, N.; Haziqah Che Marzuki, N.; Aziah Buang, N.; Huyop, F.; Abdul Wahab, R. An Overview of Technologies for Immobilization of Enzymes and Surface Analysis Techniques for Immobilized Enzymes. Biotechnol. Biotechnol. Equip. 2015, 29, 205220.

(49) Lou, D.; Ji, L.; Fan, L.; Ji, Y.; Gu, N.; Zhang, Y. Antibody-Oriented Strategy and Mechanism for the Preparation of Fluorescent Nanoprobes for Fast and Sensitive Immunodetection. Langmuir 2019, 35, 4860-4867.

(50) Verbeke, K.; Gils, A.; Declerck, P. J. Inhibition of Plasminogen Activator Inhibitor-1: Antibody Fragments and Their Unique Sequences as a Tool for the Development of Profibrinolytic Drugs. J. Thromb. Haemost. 2004, 2 (2), 298-305. https://doi.org/10.1111/j.1538-7933.2004.00583.x.

(51) Thompson, L. C.; Goswami, S.; Peterson, C. B. Metals Affect the Structure and Activity of Human Plasminogen Activator Inhibitor-1. II. Binding Affinity and Conformational Changes. Protein Sci. 2011, 20 (2), 366-378. https://doi.org/10.1002/pro.567.

(52) Gils, A.; Lester, ; Meissenheimer, M.; Compernolle, G.; Declerck, P. J.; Gils, A. Species-Dependent Molecular Drug Targets in Plasminogen Activator Inhibitor-1 (PAI-1). J. Thromb. Haemost. 2009, No. 02 609-610.

(53) ) Sillen M, Weeks SD, Zhou X, Komissarov AA, Florova G, Idell S, Strelkov SV, Declerck PJ. Molecular mechanism of two nanobodies that inhibit PAI-1 activity reveals a modulation at distinct stages of the PAI-1/plasminogen activator interaction. J. Thromb. Haemost 2019 (in press)

(54) Booth, N. A.; Simpson, A. J.; Croll, A.; Bennett, B.; MacGregor, I. R Plasminogen Activator Inhibitor (PAI-1) in Plasma and Platelets. Br. J. Haematol. 1988, 70, 327-333. 


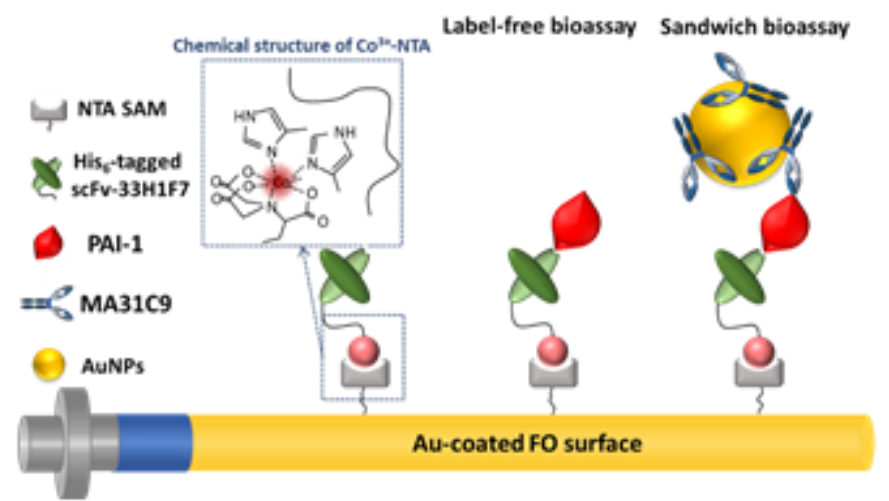

For TOC only 